DOI: 10.1515/awutp -2015-0015

\title{
NEW RESULTS ON CONSISTENT COUPLING BETWEEN BF MODELS AND DUAL LINEARIZED GRAVITY
}

\author{
C. Bizdadea, S. O. Saliu, L. Stanciu-Oprean
}

Faculty of Physics, University of Craiova 13 Al. I. Cuza Str., Craiova 200585, Romania

\section{Article Info \\ Received: 29 December 2011 \\ Accepted: 31 January 2012}

Keywords: BRST symmetry, consistent interactions, topological BF models, linearized gravity.

\begin{abstract}
Given the recent renewed interest in alternative description of gravity theories in terms of topological BF models (possibly with extra-constraints) as well as in dual formulations of linearized gravity (DFLG), all consistent interactions in $\mathrm{D}=6$ involving an Abelian $\mathrm{BF}$ model with a maximal field spectrum and the DFLG via a massless tensor field with the mixed symmetry of the type $(3,1)$ are considered.
\end{abstract}

\section{Introduction}

In this paper we construct all consistent interactions in $D=6$ spacetime dimensions between a massless tensor gauge field with the mixed symmetry of a two-column Young diagram of the type $(3,1)$ and an Abelian BF model with a maximal field spectrum (a scalar field, two sorts of 1-forms, two types of 2-forms and a 3-form).

The subject approached in this paper is important in theoretical physics due on the one hand to the fact that topological field theories [1], in particular BF models (with some extra constraints), are involved in the reformulation of general relativity in higher dimensions and supergravity in Ashtekar formalism [2]-[5], and, on the other hand, mixed symmetry-type tensor fields [6]-[9] furnish viable DFLGs. In view of this, our main task is the construction of consistent couplings in $\mathrm{D}=6$ between a specific DFLG and a topological BF model.

Our analysis relies on the deformation of the solution to the master equation [10] by means of cohomological techniques with the help of the local BRST cohomology [11]-[12]. The self-interactions in the $(3,1)$ sector have been investigated in [13]. Under the hypotheses of analyticity in the coupling constant, spacetime locality, Lorentz covariance, and Poincaré invariance of the deformations, combined with the preservation of the number of derivatives 
on each field, we find a deformation of the solution to the master equation that provides nontrivial cross-couplings. The same problem has been investigated previously [14] at the level of cross-couplings in $D=5$ between the DFLG based on a tensor field with the mixed symmetry $(2,1)$ and a topological BF model with a maximal field spectrum.

\section{The free theory}

The starting point is a free theory in $D=6$, whose Lagrangian action is written as the sum between the Lagrangian action of an Abelian BF model with a maximal field spectrum (a single scalar field $\varphi$, two types of one-forms $H^{\mu}$ and $A_{\mu}$, two kinds of two-forms $B^{\mu v}$ and $\phi_{\mu \nu}$, and a three-form $K^{\mu \nu \rho}$ ) and the Lagrangian action of a free, massless tensor field with the mixed symmetry $(3,1) t_{\mu v \rho \alpha}$ (meaning it is antisymmetric in its first three indices $t_{\mu \nu \rho \alpha}=-t_{\nu \mu \rho \alpha \alpha}$ and fulfills the identity $t_{[\mu \nu \rho \alpha]} \equiv 0$ )

$$
S_{0}^{\mathrm{L}}=\int \mathrm{d}^{6} x\left[H_{\mu} \partial^{\mu} \varphi+\frac{1}{2} B^{\mu v} \partial_{[\mu} A_{v]}+\frac{1}{3} K^{\mu v \rho} \partial_{[\mu} \phi_{v \rho]}-\frac{1}{48}\left(F_{\mu v \rho \jmath \mid \alpha} F^{\mu v \rho \lambda \mid \alpha}-4 F_{\mu v \rho} F^{\mu v \rho}\right)\right],
$$

where $F_{\mu \nu \rho \lambda \alpha}=\partial_{[\mu} t_{\nu \rho \lambda] \mid \alpha}$ and $F_{\mu \nu \rho}=\sigma^{\lambda \alpha} F_{\mu \nu \rho \jmath \alpha}$.

Everywhere in this paper we use the Minkowski metric $\sigma_{\mu v}=\sigma^{\mu v}=\operatorname{diag}(-,+\cdots+)$ and also the 6-dimensional Levi-Civita tensor $\varepsilon_{\mu v \rho \imath \sigma \theta}$ valued like $\varepsilon_{012345}=-\varepsilon^{012345}=-1$.

The Lagrangian action is invariant under the gauge transformations

$$
\begin{gathered}
\delta_{\Omega} \varphi=0, \quad \delta_{\Omega} H^{\mu}=2 \partial_{v} \varepsilon^{\mu v}, \quad \delta_{\Omega} A^{\mu}=\partial^{\mu} \varepsilon, \quad \delta_{\Omega} \phi_{\mu v}=\partial_{[\mu} \xi_{v]}, \quad \delta_{\Omega} B^{\mu v}=-3 \partial_{\rho} \varepsilon^{\mu v \rho}, \\
\delta_{\Omega} K^{\mu v \rho}=4 \partial_{\lambda} \xi^{\mu v \rho \lambda}, \quad \delta_{\Omega} t_{\mu v \rho \alpha}=3 \partial_{\alpha} \theta_{\mu v \rho}+\partial_{[\mu} \theta_{v \rho] \alpha}+\partial_{[\mu} \chi_{v \rho] \alpha},
\end{gathered}
$$

where all the gauge parameters are bosonic, with $\varepsilon^{\mu v}, \varepsilon^{\mu v \rho}, \xi^{\mu \nu \rho \lambda}, \theta_{\mu \nu \rho}$ completely antisymetric and $\chi_{\mu \eta \rho}$ a tensor gauge parameter with the mixed symmetry $(2,1)$.

This generating set of gauge transformations is Abelian and off-shell, fourth-order reducible. In order to construct the BRST symmetry of this free theory, we introduce the field, ghost and antifield spectra. The fermionic ghosts $\eta^{\alpha_{1}}=\left(C^{\mu v}, \eta^{\mu v \rho}, \Gamma^{\mu v \rho \lambda}, C_{\mu}, \mathrm{A}_{\mu v \rho}, S_{v \rho \mid \alpha}, \eta\right)$ correspond to the bosonic gauge parameters, the bosonic ghosts $\eta^{\alpha_{2}}=\left(C^{\mu v \rho}, \eta^{\mu v \rho \lambda}, \Gamma^{\mu v \rho \lambda \sigma}, C, \mathrm{~A}_{\mu v}, S_{v \alpha}\right)$ to the first-order reducibility, the

fermionic ghosts $\eta^{\alpha_{3}}=\left(C^{\mu \nu \rho \lambda}, \eta^{\mu \nu \rho \lambda \sigma}, \Gamma^{\mu v \rho \lambda \sigma \theta}, \mathrm{A}_{v}\right)$ to the second-order reducibility, the 
bosonic ghosts $\eta^{\alpha_{4}}=\left(C^{\mu v \rho \lambda \sigma}, \eta^{\mu v \rho \lambda \sigma \theta}\right)$ to to the third-order reducibility and finally the fermionic ghosts $\eta^{\alpha_{5}}=\left(C^{\mu \nu \rho \lambda \sigma \theta}\right)$ to the fourth-order reducibility. For each field/ghost we introduce the corresponding antifield and denote it by a star superscript.

Since both the gauge generators and the reducibility functions for this model are fieldindependent, it follows that the BRST differential $s$ reduces to $s=\delta+\gamma$, where $\delta$ is the Koszul-Tate differential and $\gamma$ is the exterior differential along the gauge orbits.

The BRST differential has a canonical action $s \bullet=(\bullet, S)$, where its canonical generator satisfies the classical master equation, $(S, S)=0$. The symbol (,) denotes the antibracket, defined by decreeing the fields/ghosts conjugated with the corresponding antifields. In the case of the free theory under discussion, the solution to the master equation takes the form

$$
\begin{aligned}
& S=S_{0}^{\mathrm{L}}+\int \mathrm{d}^{6} x\left[2 H_{\mu}^{*} \partial_{\nu} C^{\mu v}+A^{* \mu} \partial_{\mu} \eta-3 B_{\mu \nu}^{*} \partial_{\rho} \eta^{\mu \nu \rho}+\phi^{* \mu \nu} \partial_{[\mu} C_{\nu]}+4 K_{\mu \nu \rho}^{*} \partial_{\lambda} \Gamma^{\mu \nu \rho \lambda}-3 C_{\mu \nu}^{*} \partial_{\rho} C^{\mu \nu \rho}\right. \\
& +4 \eta_{\mu v \rho}^{*} \partial_{\lambda} \eta^{\mu \nu \rho \lambda}+C^{* \mu} \partial_{\mu} C+t^{* \mu v \rho \alpha \alpha}\left(\partial_{[\mu} S_{v \rho] \alpha}+\partial_{[\mu} A_{v \rho] \alpha}+3 \partial_{\alpha} A_{\mu v \rho}\right)-5 \Gamma_{\mu v \rho \lambda}^{*} \partial_{\sigma} \Gamma^{\mu v \rho \lambda \sigma} \\
& -\frac{1}{2} \mathrm{~A}^{* \mu v \rho} \partial_{[\mu} \mathrm{A}_{v \rho]}+S^{* \mu \nu \alpha}\left(2 \partial_{\alpha} \mathrm{A}_{\mu v}-\partial_{[\mu} \mathrm{A}_{v] \alpha}+\partial_{[\mu} S_{v] \alpha}\right)-3 S^{* \mu v} \partial_{(\mu} \mathrm{A}_{v)} \\
& +\mathrm{A}^{* \mu \nu} \partial_{[\mu} \mathrm{A}_{v]}+4 C_{\mu v \rho}^{*} \partial_{\lambda} C^{\mu v \rho \lambda}-5 \eta_{\mu v \rho \lambda}^{*} \partial_{\sigma} \eta^{\mu v \rho \lambda \sigma}+6 \Gamma_{\mu v \rho \lambda \sigma}^{*} \partial_{\theta} \Gamma^{\mu v \rho \lambda \sigma \theta} \\
& \left.-5 C_{\mu \nu \rho \lambda}^{*} \partial_{\sigma} C^{\mu \nu \rho \lambda \sigma}+6 C_{\mu \nu \rho \lambda \sigma}^{*} \partial_{\theta} C^{\mu v \rho \lambda \sigma \theta}+6 \eta_{\mu v \rho \lambda \sigma}^{*} \partial_{\theta} \eta^{\mu \nu \rho \lambda \sigma \theta}\right] .
\end{aligned}
$$

The solution to the master equation contains all the information on the gauge structure of the theory. In our case the solution to the master equation breaks into terms with the antighost number ranging from zero to five. The part with the antighost number equal to zero is nothing but the Lagrangian action of the gauge model under study. The components of antighost number equal to one are always proportional with the gauge generators. The absence of terms linear in the antighost number two antifields and quadratic in the pure ghost number one ghosts, in our case, shows that the gauge transformations are Abelian. The terms from (4) with higher antighost numbers give us information on the reducibility functions.

\section{Deformation of the master equation}

We can reformulate the problem of constructing consistent interactions among the fields of the theory as a deformation problem of the solution to the master equation corresponding to the "free" theory. If a consistent interacting gauge theory can be constructed, then the solution $S$ to the master equation associated with the "free" theory, can be deformed into a solution $\bar{S}$ for the interacting theory

$$
S \rightarrow \bar{S}=S+g S_{1}+g^{2} S_{2}+\cdots,
$$




$$
(\bar{S}, \bar{S})=0
$$

such that both the ghost and antifield spectra of the initial theory are preserved. Projecting eq. (6) on various powers in the coupling constant, we find that the equivalent tower of equations has to be satisfied

$$
\begin{gathered}
(S, S)=0, \\
2\left(S_{1}, S\right)=0, \\
2\left(S_{2}, S\right)+\left(S_{1}, S_{1}\right)=0, \\
\left(S_{3}, S\right)+\left(S_{1}, S_{2}\right)=0,
\end{gathered}
$$

Eq. (7) is satisfied by hypothesis, since $S$ is the solution for the master equation for the starting theory. The construction of consistent interactions becomes equivalent to solving Eqs. (8)-(10), etc.

\section{Consistent Lagrangian interactions}

Using the method described in the previous section, we are able to determine the Lagrangian action for the interacting theory as

$$
\begin{gathered}
S_{\text {int }}^{\mathrm{L}}\left[\Phi^{\alpha_{0}}\right]=\int \mathrm{d}^{6} x\left[H_{\mu}\left(\partial^{\mu} \varphi-g W_{1} A^{\mu}\right)+\frac{1}{2} B^{\mu v}\left(\partial_{[\mu} A_{\nu]}-2 g W_{2} \phi_{\mu \nu}\right)+\frac{1}{3} K^{\mu v \rho}\left(\partial_{[\mu} \phi_{v \rho]}+3 g W_{3} A_{[\mu} \phi_{\nu \rho]}\right)\right. \\
+g M(\varphi)-\frac{1}{8} g \varepsilon^{\mu v \rho \lambda \sigma \theta} W_{4} \phi_{\mu \nu} \phi_{\rho \lambda} \phi_{\sigma \theta}-\frac{1}{48}\left(F_{\mu \nu \rho \lambda \alpha} F^{\mu v \rho \lambda \alpha}-4 F_{\mu v \rho} F^{\mu v \rho}\right) \\
\left.+\frac{2}{3} g F_{\mu \nu \rho}\left(\frac{1}{6 !} U_{1} \widetilde{K}^{\mu v \rho}+\frac{1}{6} U_{3} A^{[\mu} \phi^{\nu \rho]}\right)+2 g^{2}\left(\frac{1}{6 !} U_{1} \widetilde{K}^{\mu v \rho}+\frac{1}{6} U_{3} A^{[\mu} \phi^{\nu \rho]}\right)\left(\frac{1}{6 !} U_{1} \widetilde{K}_{\mu \nu \rho}+\frac{1}{6} U_{3} A_{[\mu} \phi_{v \rho]}\right)\right] .
\end{gathered}
$$

Meanwhile, we find the gauge transformations that leave invariant the above action, namely,

$$
\begin{aligned}
& \bar{\delta}_{\Omega} \varphi=g W_{1} \varepsilon, \quad \bar{\delta}_{\Omega} A^{\mu}=\partial^{\mu} \varepsilon+2 g W_{2} \xi^{\mu} \\
& \bar{\delta}_{\Omega} H^{\mu}=2 D_{v} \varepsilon^{\mu v}+g\left(3 \frac{d W_{3}}{d \varphi} K^{\mu v \rho} \phi_{v \rho}-\frac{d W_{1}}{d \varphi} H^{\mu}\right) \varepsilon+2 g\left(3 \frac{d W_{3}}{d \varphi} A_{\rho} K^{\mu v \rho}-\frac{d W_{2}}{d \varphi} B^{\mu v}\right) \xi_{v} \\
& +3 g \frac{d W_{2}}{d \varphi} \phi_{\nu \rho} \varepsilon^{\mu v \rho}-12 g \frac{d W_{3}}{d \varphi} A_{v} \phi_{\rho \lambda} \xi^{\mu v \rho \lambda}-\frac{3}{4} g \frac{d W_{4}}{d \varphi} \varepsilon^{\mu \nu \rho \lambda \sigma \theta} \phi_{v \rho} \phi_{\lambda \sigma} \xi_{\theta}-g \frac{d U_{3}}{d \varphi} A_{v} \phi_{\rho \lambda} \partial^{[\mu} \theta^{v \rho \lambda]} \\
& -g F^{\mu \nu \rho}\left(\frac{1}{3 \cdot 6 !} \frac{d U_{1}}{d \varphi} \varepsilon_{\nu \rho \lambda \sigma \theta \varepsilon} \xi^{\lambda \sigma \theta \varepsilon}+\frac{2}{3} \frac{d U_{3}}{d \varphi} A_{\nu} \xi_{\rho}-\frac{1}{3} \frac{d U_{3}}{d \varphi} \phi_{\nu \rho} \varepsilon\right)-\frac{1}{3 \cdot 6 !} g \frac{d U_{1}}{d \varphi} \varepsilon_{v \rho \lambda \sigma \theta \varepsilon} K^{\sigma \theta \varepsilon} \partial^{[\mu} \theta^{v \rho \lambda]} \\
& -\frac{1}{9.5 !} g^{2} \varepsilon^{\mu \nu \rho \lambda \sigma \theta} K_{\lambda \sigma \theta} U_{1} \frac{d U_{3}}{d \varphi}\left(A_{v} \xi_{\rho}-\frac{1}{2} \phi_{v \rho} \varepsilon\right)+\frac{1}{45 \cdot 6 !} g^{2} U_{1} \frac{d U_{1}}{d \varphi} K_{v \rho \lambda} \xi^{\mu v \rho \lambda} \\
& +\frac{1}{3} g^{2} U_{3} A^{[\mu} \phi^{v \rho]}\left(\frac{d U_{3}}{d \varphi} \phi_{v \rho} \varepsilon-2 \frac{d U_{3}}{d \varphi} A_{v} \xi_{\rho}-\frac{1}{6 !} \frac{d U_{1}}{d \varphi} \varepsilon_{v \rho \lambda \sigma \theta \varepsilon} \xi^{\lambda \sigma \theta \varepsilon}\right)
\end{aligned}
$$




$$
\begin{aligned}
& \bar{\delta}_{\Omega} B^{\mu v}=-3 \partial_{\rho} \varepsilon^{\mu v \rho}+2 g W_{1} \varepsilon^{\mu v}-6 g\left(2 W_{3} \phi_{\rho \lambda} \xi^{\mu v \rho \lambda}+W_{3} K^{\mu v \rho} \xi_{\rho}\right)-g U_{3} \phi^{\rho \lambda} \partial_{[\mu} \theta_{v \rho \lambda]} \\
& -\frac{2}{3} g U_{3}\left(F^{\mu \nu \rho}+g U_{3} A^{[\mu} \phi^{\nu \rho]}+\frac{1}{6 !} g U_{1} \varepsilon^{\mu \nu \rho \gamma \sigma \theta} K_{\lambda \sigma \theta}\right) \xi_{\rho}, \\
& \bar{\delta}_{\Omega} \phi_{\mu v}=D_{[\mu}^{(+)} \xi_{v]}-3 g W_{3} \phi_{\mu \nu} \varepsilon-\frac{1}{6 !} g U_{1} \varepsilon_{\mu \nu \rho \gamma \sigma \theta} \partial^{\rho} \theta^{\lambda \sigma \theta}, \\
& \bar{\delta}_{\Omega} K^{\mu v \rho}=4 D_{\lambda}^{(-)} \xi^{\mu v \rho \imath}+3 g W_{2} \varepsilon^{\mu v \rho}+3 g W_{3} K^{\mu v \rho} \varepsilon-\frac{3}{2} g W_{4} \varepsilon^{\mu v \rho r \sigma \theta} \phi_{\lambda \sigma} \xi_{\theta} \\
& +\frac{1}{3} g U_{3}\left(F^{\mu \nu \rho}+g U_{3} A^{[\mu} \phi^{\nu \rho]}+\frac{1}{6 !} g U_{1} \varepsilon^{\mu \nu \rho \lambda \sigma \theta} K_{\lambda \sigma \theta}\right) \varepsilon-g U_{3} A_{\lambda} \partial^{[\mu} \theta^{v \rho \lambda]}, \\
& \bar{\delta}_{\Omega} t_{\mu \nu \rho \alpha \alpha}=3 \partial_{\alpha} \theta_{\mu v \rho}+\partial_{[\mu} \theta_{v \rho] \alpha}+\partial_{[\mu} \chi_{v \rho] \alpha}+\frac{1}{3} g U_{3} \sigma_{\alpha[\mu} A_{\nu} \xi_{\rho]}-\frac{1}{3} g U_{3} \sigma_{\alpha[\mu} \phi_{v \rho]} \varepsilon \\
& +\frac{1}{3.6 !} g U_{1} \sigma_{\alpha[\mu} \varepsilon_{v \rho] \lambda \sigma \theta \varepsilon} \xi^{\lambda \sigma \theta \varepsilon}
\end{aligned}
$$

where we denoted $D_{v}=\partial_{v}+g \frac{d W_{1}}{d \varphi} A_{v}$ and $D^{( \pm)}{ }_{v}=\partial_{v} \pm g W_{3} A_{v}$.

The Lagrangian action contains only interaction vertices of order one and respectively two in the coupling constant. It is interesting to note that there appear some self-interactions in the BF sector that are strictly due to the presence of the tensor field with the mixed symmetry $(3,1)$ (in its absence they all vanish). The gauge transformations of all fields are deformed. The gauge algebra becomes open and the reducibility relations hold on-shell.

The functions $\left(W_{i}\right)_{i=\overline{1,4}}, M(\varphi)$ and $\left(U_{i}\right)_{i=1,3}$ that appear in (11) are smooth functions depending only on the undifferentiated scalar field, restricted to satisfy the equations

$$
\begin{gathered}
\frac{d M(\varphi)}{d \varphi} W_{1}(\varphi)=0, \quad W_{1}(\varphi) W_{2}(\varphi)=0, \quad W_{2}(\varphi) W_{3}(\varphi)=0, \\
W_{1}(\varphi) \frac{d W_{2}(\varphi)}{d \varphi}=0, \quad W_{3}(\varphi) W_{4}(\varphi)=0 \quad W_{1}(\varphi) \frac{d W_{4}(\varphi)}{d \varphi}=0, \\
3 W_{3}(\varphi) U_{1}(\varphi)+W_{1}(\varphi) \frac{d U_{1}(\varphi)}{d \varphi}=0, \\
W_{2}(\varphi) U_{1}(\varphi)=0, \quad W_{4}(\varphi) U_{1}(\varphi)-160 W_{2}(\varphi) U_{3}(\varphi)=0 .
\end{gathered}
$$

We are only interested in the solutions to equations (18) -(21) that describe crosscouplings, so only in the solutions with at least one of the functions $U_{1}$ or $U_{2}$ non-vanishing. There are two different types of such solutions to (18)-(21). The first type is described by the choice

$$
W_{1}(\varphi)=W_{2}(\varphi)=W_{3}(\varphi)=W_{4}(\varphi)=0
$$

with $U_{1}(\varphi), U_{3}(\varphi)$ and $M(\varphi)$ arbitrary smooth functions of $\varphi$.

The second type of solution in given by 


$$
W_{2}(\varphi)=W_{4}(\varphi)=M(\varphi)=0 \text { and } U_{1}(\varphi) \neq 0,
$$

$U_{3}(\varphi)$ is an arbitrary smooth function and $W_{3}(\varphi)$ and $U_{1}(\varphi)$ have to satisfy

$$
3 W_{3}(\varphi) U_{1}(\varphi)+W_{1}(\varphi) \frac{d U_{1}(\varphi)}{d \varphi}=0
$$

whose solution is given by

$$
U_{1}(\varphi)=\exp \left(-\int \frac{3 W_{3}}{W_{1}} d \varphi\right)
$$

\section{Acknowledgements}

One of the authors (L.S.O.) acknowledges partial support from the strategic grant POSDRU/88/1.5/S/49516, Project ID 49516 (2009), co-financed by the European Social Fund -- Investing in People, within the Sectoral Operational Programme Human Resources Development 2007 - 2013.

\section{References}

[1] D. Birmingham, M. Blau, M. Rakowski, G. Thompson, Phys. Rept., 209 (1991) 129;

[2] K. Ezawa, Prog. Theor. Phys., 95 (1996) 863;

[3] L. Freidel, K. Krasnov, R. Puzio, Adv. Theor. Math. Phys., 3 (1999) 1289;

[4] L. Smolin, Phys. Rev. D, 61 (2000) 084007;

[5] Y. Ling, L. Smolin, Phys. Rev. D, 63 (2001) 064010;

[6] T. Curtright, P. G. O. Freund, Nucl. Phys. B, 172 (1980) 413;

[7] T. Curtright, Phys. Lett. B, 165 (1985) 304;

[8] C. S. Aulakh, I. G. Koh, S. Ouvry, Phys. Lett. B, 173 (1986) 284;

[9] J. M. Labastida, T. R. Morris, Phys. Lett. B, 180 (1986) 101;

[10] G. Barnich, M. Henneaux, Phys. Lett. B, 311 (1993) 123;

[11] G. Barnich, F. Brandt, M. Henneaux, Commun. Math. Phys., 174 (1995) 93;

[12] G. Barnich, F. Brandt, M. Henneaux, Commun. Math. Phys., 174 (1995) 57;

[13] C. Bizdadea, C. C. Ciobîrcă, E. M. Cioroianu, I. Negru, S. O. Saliu, S. C. Săraru, J. High Energy Phys. JHEP, 10 (2003) 019;

[14] C. Bizdadea, E. M. Cioroianu, A. Danehkar, M. Iordache, S. O. Saliu, S. C. Săraru, Eur. Phys. J. C, 63 (2009) 491. 